Received: 2015.08 .06

Accepted: 2015.09.22

Published: 2016.01 .19

Authors' Contribution: Study Design A Data Collection B Statistical Analysis C Data Interpretation D Manuscript Preparation E Literature Search F Funds Collection G

Corresponding Author: Source of support:

\title{
Reliability of an Electronic Inspiratory Loading Device for Assessing Pulmonary Function in Post-Stroke Patients
}

Kyeong-Bong Lee

Min-Kyu Kim

Ju-Ri Jeong

Wan-Hee Lee
Graduate School of Physical Therapy, Sahmyook University, Seoul, Republic of Korea

Background: The purpose of this study was to examine the inter- and intra-rater reliability of an electronic inspiratory loading device for the assessment of pulmonary functions: maximum inspiratory pressure, peak inspiratory flow, and vital capacity.

Material/Methods

Wan-Hee Lee, e-mail: whlee@syu.ac.kr

Departmental sources

Subjects were 50 patient volunteers in a rehabilitation hospital who had experienced their first episode of unilateral stroke with hemiparesis during the previous 6 months (26 men, 24 women; mean age [ \pm SD], 55.96 [ \pm 12.81 ] years), with no use of medications that could induce drowsiness, evidence of restrictive lung disease, history of asthma, use of psychotropic drugs, or alcohol consumption habit.

Maximum inspiratory pressure, peak inspiratory flow, and vital capacity for pulmonary functions were assessed using an electronic inspiratory loading device (PowerBreathe, K5, 2010) by 2 examiners, with patients in an unassisted sitting position, and 1 examiner re-assessed with same patients at the same time of a day after 1 week. Intra-class correlation coefficients were used to assess reliability.

Results: Intra-rater reliability ranged from intra-class correlation coefficients (ICCs) $=0.959$ to 0.986 in variables. For the inter-rater reliability between 2 examiners, the ICCs ranged from 0.933 to 0.985 . Intra-rater and inter-rater reliability were good in variables (maximal inspiratory pressure, peak inspiratory flow, and vital capacity).

Conclusions: The intra- and inter-examiner reliability of the pulmonary function measurements, maximum inspiratory pressure, peak inspiratory flow, and vital capacity, for the post-stroke patients was very high. The results suggest that the electronic inspiratory loading device would be useful for clinical rehabilitative assessment of pulmonary function.

MeSH Keywords: Inhalation • Reproducibility of Results • Respiratory Function Tests • Stroke

Full-text PDF: http://www.medscimonit.com/abstract/index/idArt/895573

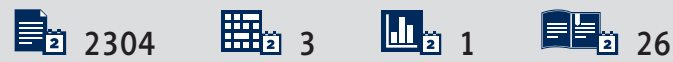




\section{Background}

Respiratory dysfunction in stroke patients is caused by weakening of the trunk and respiratory muscles and reduced physical activity due to loss of normal postural control resulting from a lesion in the central nervous system [1,2]. Because weakening of respiratory muscles decreases exercise endurance, functional locomotion, and independent walking ability [3], strengthening of respiratory muscle plays an important role in the functional recovery of post-stroke patients.

Post-stroke patients have partial or total weakening of the diaphragm, intercostal, and abdominal muscles on the affected side $[4,5]$. There is also a significant decrease in maximal respiratory pressure in post-stroke patients compared with that in healthy individuals [5]. About $40 \%$ of stroke patients show a reduction in diaphragm displacement and a decrease in lung function to $50 \%$ of the age-expected values $[6,7]$. Both residual volume (RV) and vital capacity (VC) are affected, leading to respiratory muscle weakness. Recent research reported that inspiratory muscle training is effective in improving activities of daily living, walking ability, quality of life, respiratory muscle strength, and endurance in stroke patients $[8,9]$. Thus, assessment of respiratory muscle strength may be useful to monitor the improvement in patient health over time, identify those at risk of hypoventilation, and determine the impact of respiratory muscle training, and can assess respiratory muscle weakness and quantify its severity [10].

Maximal inspiratory pressure (MIP) is a widely used, clinically relevant indicator of the effect of respiratory training. The MIP is generated during the Müller maneuver, which involves a forced expiration, followed by an attempt at inspiration made with the mouth and nose closed, which leads to a negative pressure in the chest and lungs. It is the volitional force output of the inspiratory muscles working in synergy, and is an established and reliable measure of global inspiratory muscle strength $[11,12]$.

Previous studies have reported that respiratory muscle training improves muscle function in acute stroke patients [13] and inspiratory muscular weakness is evident in chronic stroke survivors [14]. However, data on the reliability of measurements used in inspiratory muscle training and respiratory functions for post-stroke patients are lacking. Peak inspiratory flow (PIF), at a given volume, depends on the airway caliber as well as the strength and speed of shortening of the inspiratory muscles [15]. The muscle-training device is an electronic inspiratory loading device, and can provide automatically processed information for the MIP, PIF, and VC during loaded breathing tasks.

The purpose of this study was to determine the intra- and inter-reliability for measurement of pulmonary function values,
Table 1. General characteristics of subjects.

\begin{tabular}{|cc|}
\hline \multicolumn{1}{c}{ Characteristic } & Mean $\mathbf{\text { SD }}$ \\
\hline Gender, male/female & $26 / 24$ \\
\hline Age, years & $55.96 \pm 12.81$ \\
\hline Height, cm & $164.77 \pm 6.97$ \\
\hline Weight, kg & $63.11 \pm 10.53$ \\
\hline BMI, kg/m ${ }^{2}$ & $23.12 \pm 2.68$ \\
\hline Affected side, left/right & $29 / 21$ \\
\hline Duration, days & $228.66 \pm 150.82$ \\
\hline
\end{tabular}

SD - standard deviation; BMI - body mass index.

MIP, PIF and VC. Our hypothesis is that the electronic inspiratory loading device is sufficiently reliable for pulmonary function tests in post-stroke patients.

\section{Material and Methods}

\section{Subjects}

Fifty post-stroke patients from the M Rehabilitation Hospital in Seoul, Republic of Korea, volunteered and participated in this study. A physical assessment and interview were initially conducted with all individuals to collect anthropometric and demographic data (gender, age, height, weight, body mass index [BMI], affected side, onset time of stroke, and use of medications). A summary of the general characteristics of subjects is provided in Table 1. A questionnaire was administered to gather information related to the work environment, smoking history, recent respiratory infections, and the presence or absence of lung and heart disease. The data were used as parameters for both the analyses and interpretation of the pulmonary function tests.

The inclusion criteria were as follows: no history of cardiovascular or respiratory problems, first episode of unilateral stroke with hemiparesis during the previous 6 months, mini mental status evaluation score $>24$, no facial palsy, no receptive aphasia, and no prior thoracic or abdominal surgery. The exclusion criteria were as follows: use of medications that could affect neuromuscular control or provoke drowsiness, evidence of restrictive lung disease based on lung volumes, history of asthma, and use of psychotropic drugs or alcohol abuse. The study's protocol was approved by the institutional review board of the Sahmyook University in Seoul and participants gave signed consent after receiving verbal and written information about the study. 


\section{Examiners}

Two physical therapists participated as examiners in the reliability analysis. Examiner 1 had been a physical therapist for 2 years, and Examiner 2 had been a physical therapist for 12 years. Neither of the examiners had evaluated pulmonary function using the device in their rehabilitation clinic. Before beginning the study, both examiners underwent 24 hours of specific training in educational and experimental methods for patients, with a professional instructor (Ph.D.) experienced with the measurement protocol used in this study.

\section{Study design}

The examiners tested all subjects during 2 separate sessions. Two measurements were performed on the same day to assess inter-rater reliability and to ensure that the pulmonary function parameters were unchanged [16]. After a week, 1 examiner measured the variables again at the same time of a day to assess intra-rater reliability. The measurements were performed in exactly the same way.

\section{Experimental procedure}

The electronic inspiratory loading device (PowerBreathe K5, 2010, $\mathrm{HaB}$ International Ltd, UK) was used to provide automatically processed information on pulmonary function (MIP, PIF, and VC) during loaded breathing tasks. Inspiratory muscle strength was assessed by measuring the MIP at RV [17]. Subjects were comfortably seated in a chair with their feet on the ground, their back unsupported, and their trunk at a $90^{\circ}$ angle to their hips. To record MIP, participants were instructed to perform inspirations against an obstructed airway at near-RV. A conventional mouthpiece and nose clip were used to prevent leakage of air. The pressure, which was sustained for 2-3 seconds, was recorded. Subjects could choose their own breathing frequency, but were instructed to perform forceful and deep inspirations followed by complete expirations. Complete expiration was indicated by an acoustic signal provided by the handheld loading device upon cessation of flow. Subjects were given strong verbal encouragement but no visual feedback. Subjects performed a minimum of 3 efforts and the value used was the best of these. Subjects were allowed to practice twice and immediately afterwards were asked to repeat the trial until 3 acceptable measurements were obtained [18]. Measurements were considered acceptable if they were maintained, without an air leak, for duration of at least 1 second and if 2 readings were taken with a maximum difference of $10 \%$. Between the measurements, 20 minutes of break time was given to patients to minimize the bias for the MIP, PIF, and VC due to inter-rater reliability test. The highest MIP values were recorded for analyses. Signals from the pneumotachograph were captured electronically using J-Lab software version 5.22.1.50 (Cardinal Health $\mathrm{GmbH}$, Hoechberg, Germany).

\section{Statistical analysis}

To describe the intra- and inter-rater reliability of the electronic inspiratory loading device for pulmonary functions, intraclass correlation coefficients (ICCS) were calculated for MIP, PIF, and VC. ICCs of the type $(3,1)$ were used to evaluate the inter-rater reliability and type $(1,1)$ for intra-rater reliability. Bland-Altman plots and $95 \%$ confidence intervals (Cls) are given to provide a visual representation of pressure and volume and to determine the agreement between the 2 measurements [19]. ICC and Bland-Altman plots have been advocated as the statistical methods of choice in reliability studies [20]. ICCs $\leq 0.25$ were considered poor, 0.26-0.49 low, 0.50-0.69 moderate, $0.70-0.89$ high, and $0.90-1.00$ very high. If the slope of the scatter plot of 2 variables in Bland-Altman plot is $Y=0$, the 2 tests will have little error [19].

To assess measurement, standard error of measurement (SEM) was calculated as SD * $\sqrt{1-I C C}$. The $95 \%$ minimal detectable change (MDC) was calculated as $1.96 *$ SEM * $\sqrt{2}$ [21]. SEM and MDC are often used to determine the standard error of the measurement; their reliability ensures the accuracy and precision of the smaller observed standard error of the measured variable. Results were considered significant at $p<0.05$, and statistical analyses were performed using SPSS (version 18.0).

\section{Results}

A summary of results for intra-rater reliability between examiners for the 3 sessions is given in Table 2. The ICCs for MIP, PIF, and VC were $0.986,0.992$, and 0.959 , respectively. The measurements were therefore considered highly reliable. A summary of results for inter-rater reliability between tests for the 3 sessions performed is shown in Table 3. The ICCS for MIP, PIF, and VC were $0.984,0.985$, and 0.933 , respectively. The measurements were therefore considered highly reliable. SEM for MIP, PIF, and VC varied between 3.575 and $4.380 \mathrm{mmH}_{2} \mathrm{O}$, $0.003 \mathrm{l} / \mathrm{s}$ and $0.057 \mathrm{l} / \mathrm{s}, 0.122$ and 0.179 , respectively, and $95 \%$ minimal detectable change ranged from 4.917 to $7.370 \mathrm{~mm}$ $\mathrm{H}_{2} \mathrm{O}$ for MIP, 0.000 to $3.615 \mathrm{l} / \mathrm{s}$ for PIF, and 0.057 to $0.123 \mathrm{l} / \mathrm{s}$ for TLC. Bland-Altman plots indicated good agreement, and the plots for inter- and intra-rater reliability are shown in Figure 1.

\section{Discussion}

Inspiratory muscle training has been used to investigate cardiopulmonary function and exercise tolerance for chronic and subacute post-stroke patients [8,9]. Following a stroke, patients experience respiratory dysfunction because of reduction in vital capacity, inspiratory capacity, total lung capacity, maximum inspiratory capacity, and expiratory reserve volume [22]. 
Table 2. Intra-rater reliability between measures for the variables.

\begin{tabular}{|c|c|c|c|c|c|c|}
\hline \multirow{2}{*}{ Variables } & \multicolumn{2}{|c|}{ Mean \pm SD } & \multirow{2}{*}{ ICC } & \multirow{2}{*}{$95 \% \mathrm{Cl}$} & \multirow{2}{*}{ SEM } & \multirow{2}{*}{ MDC } \\
\hline & $1^{\text {st }}$ test & $2^{\text {nd }}$ test & & & & \\
\hline $\begin{array}{l}\text { Maximal inspiratory } \\
\text { pressure }\left(\mathrm{CmH}_{2} \mathrm{O}\right)\end{array}$ & $52.02 \pm 20.49$ & $53.52 \pm 21.06$ & .986 & $.976-.992$ & 4.380 & 7.370 \\
\hline Peak inspiratory flow $(1 / \mathrm{s})$ & $2.92 \pm 1.20$ & $2.97 \pm 1.20$ & .992 & $.986-.995$ & 0.003 & 0.011 \\
\hline Vital capacity (l) & $1.99 \pm 0.69$ & $1.99 \pm 0.58$ & .959 & $.928-.977$ & 0.122 & 0.057 \\
\hline
\end{tabular}

SD - standard deviation; ICC - intraclass correlation coefficient; $95 \% \mathrm{Cl}-95 \%$ confidence interval; SEM - standard error of the mean; MDC - minimal detectable change.

Table 3. Inter-rater reliability between examiners for the variables.

\begin{tabular}{|c|c|c|c|c|c|c|}
\hline \multirow{2}{*}{ Variables } & \multicolumn{2}{|c|}{ Mean \pm SD } & \multirow{2}{*}{ ICC } & \multirow{2}{*}{$95 \% \mathrm{Cl}$} & \multirow{2}{*}{ SEM } & \multirow{2}{*}{ MDC } \\
\hline & $\mathbf{E 1}$ & E2 & & & & \\
\hline $\begin{array}{l}\text { Maximal inspiratory } \\
\text { pressure }\left(\mathrm{cmH}_{2} \mathrm{O}\right)\end{array}$ & $52.02 \pm 20.49$ & $52.09 \pm 21.40$ & .984 & $.971-.991$ & 3.575 & 4.912 \\
\hline Peak inspiratory flow $(1 / \mathrm{s})$ & $2.92 \pm 1.20$ & $3.01 \pm 1.24$ & .985 & $.974-.992$ & 0.006 & 0.000 \\
\hline Vital capacity (l) & $1.99 \pm 0.69$ & $1.99 \pm 0.58$ & .933 & $.881-.962$ & 0.179 & 0.123 \\
\hline
\end{tabular}

E1 - examiner 1; E2 - examiner 2; SD - standard deviation; ICC - intraclass correlation coefficient; $95 \% \mathrm{Cl}$ - $95 \%$ confidence interval; SEM - standard error of the mean; MDC - minimal detectable change.

Furthermore, the efficiency of the unaffected muscles may be decreased due to instability of the chest wall and an inactive lifestyle [9]. The aim of this study was to quantify the reliability of a respiratory muscle test device for assessing pulmonary function in post-stroke patients and to determine the implications of the results. The main finding suggests that the described protocols produce reliable measurements for most of the inspiratory muscle function tests considered. The reliability of these measures supports their use in within-subject studies.

Jandt et al. studied the relationship between trunk control, respiratory muscle strength, and pulmonary function in stroke patients, and found a positive correlation between trunk control and respiratory muscle strength, particularly in the case of the expiratory muscles [2]. In a study involving individuals with multiple sclerosis, inspiratory muscle training significantly increased inspiratory muscle strength and resulted in generalized improvements in expiratory pulmonary function [23]. In addition, inspiratory muscle training has been associated with breathing retraining and was found to be feasible and effective in patients with generalized myasthenia gravis. Improvements in respiratory muscle strength, chest wall mobility, respiratory pattern, and respiratory endurance were also observed [24]. However, other researchers have argued that there is insufficient evidence to support inspiratory muscle training as an effective treatment to improve function after stroke, and no evidence relating to the safety of inspiratory muscle training [25].
Although electronic inspiratory loading devices have previously been used to train and examine pulmonary function, their reliability when used with post-stroke patients has not been reported, and their clinical use to date has been limited. The current study evaluated the intra- and inter-rater reliability of measures of MIP, PIF, and VC, and found that the electronic inspiratory loading device had very high intra-rater reliability and very high inter-rater reliability. The $95 \% \mathrm{Cls}$ for the inter- and inter-rater reliability of MIP were narrow (0.986 and 0.0.984, respectively). Similarly, the $95 \%$ Cls for the intra- and inter-rater reliability of PIF ( 0.992 and 0.985 , respectively) and VC (0.959 and 0.933 , respectively) were also narrow. The high inter- and intra-reliability for MIP, PIF, and VC suggests that the current study had high power.

Romer and McConnell investigated the inter-test reliability of non-invasive measures of respiratory muscle function, and suggested that measures of maximum inspiratory and expiratory pressure and flow all had good reliability [11]. Gosselink et al. reported the reliability of a threshold-loading device in healthy subjects and in patients with chronic obstructive pulmonary disease (COPD). In both groups, only small variations in pressure were observed despite large variations in flow. The threshold is a reliable and reproducible device for loading inspiratory muscles in patients with COPD as well as in healthy subjects, and, similar to previous studies, showed good interrater (0.881-0.992) and intra-rater (0.928-0.992) reliability [26]. 

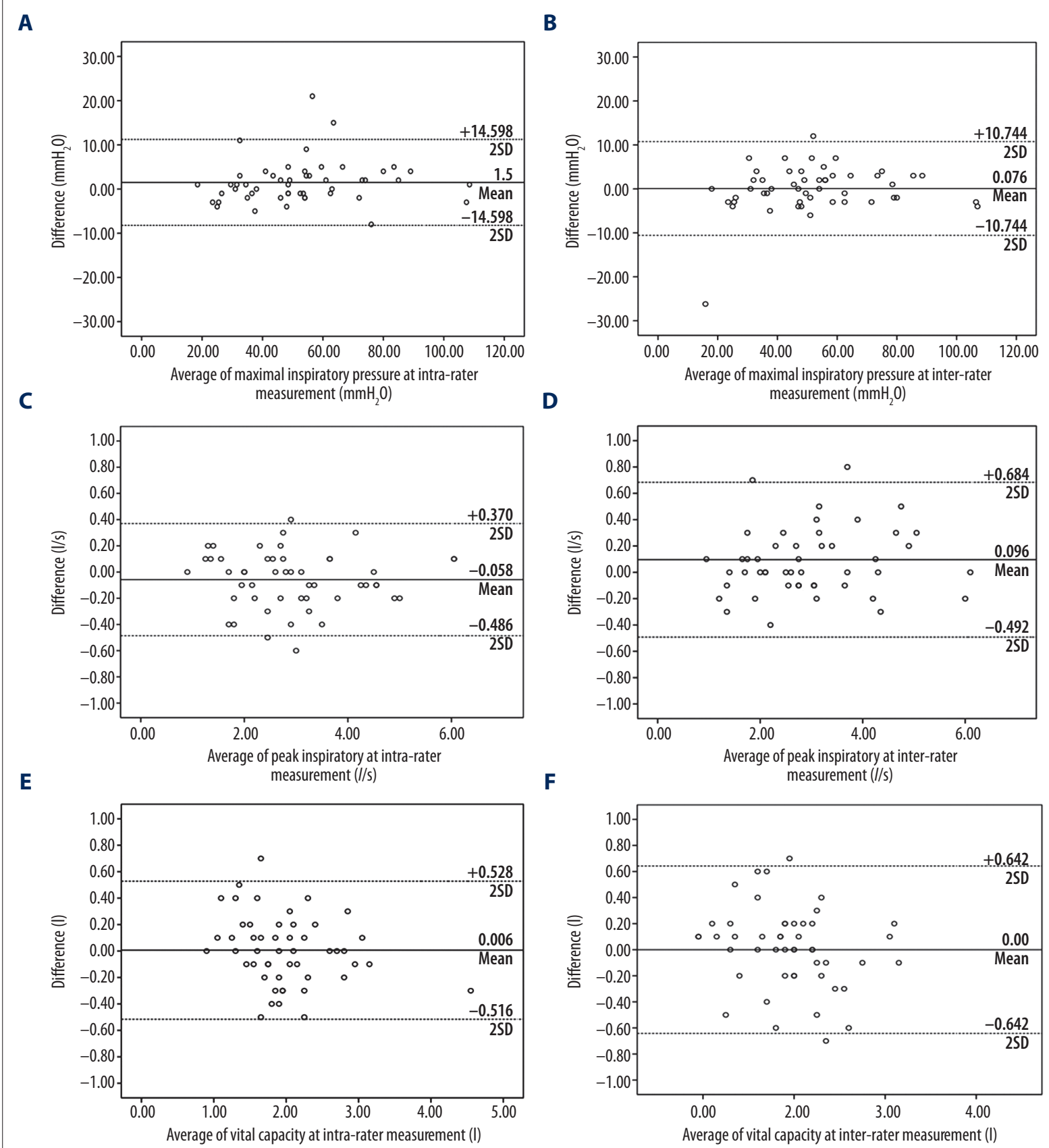

Figure 1. Bland-Altman plots illustrating the measurement difference of maximal inspiratory pressure and peak inspiratory flow volume (A): Maximal inspiratory at intra-rater measurement (B): Maximal inspiratory at inter-rater measurement (C): Peak inspiratory flow volume at intra-rater measurement (D): Peak inspiratory flow volume at inter-rater measurement (E): Vital capacity at intra-rater measurement (F): Vital capacity at inter-rater measurement

However, these reliabilities ranged from high to very high and the results showed that the procedure for the respiratory muscle test was compromised to increase reliabilities.

When SEM is less than $10 \%$ of the mean value and MDC is less than $20 \%$ of the measured value, they are reliable because the measurement error is estimated low [26]. In the present study, SEM values of MIP, PIF, and VC were less than $10 \%$ of the average measured values. Also, MDC values appeared to be less than $10 \%$ of all the 3 variables and they increased the confidence level. To use electronic inspiratory loading devices for rehabilitation in post-stroke patients, high and constant 
intra- and inter-reliability during inspiration training with patients is very important. Thus, an appropriate and coherent procedure is required.

The results of pulmonary function tests for the MIP, PIF and VC can be affected by the examiner's experience. In the current study, although the 2 examiners had clinical careers of different durations, the intra-examiner reliability was high to very high in both cases. These results show that following a consistent procedure might be adequate for the measurement of pulmonary function, despite differences in clinical experience. Therefore, the pulmonary function test device can be useful in goal-setting for post-stroke patients even when it is performed by therapists with different experience.

Our study has several limitations. First, the findings of this study are valid in post-stroke patients only and may not necessarily be generalizable to other patients. Second, the sample

\section{References:}

1. Teixeira-Salmela LF, Parreira VF, Britto RR et al: Respiratory pressures and thoracoabdominal motion in community-dwelling chronic stroke survivors. Arch Phys Med Rehabil, 2005; 86: 1974-78

2. Jandt SR, Caballero RM, Junior LA, Dias AS: Correlation between trunk control, respiratory muscle strength and spirometry in patients with stroke: an observational study. Physiother Res Int, 2011; 16: 218-24

3. Pang MY, Eng JJ, Dawson AS: Relationship between ambulatory capacity and cardiorespiratory fitness in chronic stroke: influence of stroke-specific impairments. Chest, 2005; 127: 495-501

4. Similowski T, Catala M, Rancurel G, Derenne JP: Impairment of central motor conduction to the diaphragm in stroke. Am J Respir Crit Care Med, 1996; 154: 436-41

5. Lanini B, Bianchi R, Romagnoli I et al: Chest wall kinematics in patients with hemiplegia. Am J Respir Crit Care Med, 2003; 168: 109-13

6. Khedr EM, El Shinawy O, Khedr T et al: Assessment of corticodiaphragmatic pathway and pulmonary function in acute ischemic stroke patients. Eur J Neurol, 2000; 7: 509-16

7. Larson JL, Kim MJ, Sharp JT, Larson DA: Inspiratory muscle training with a pressure threshold breathing device in patients with chronic obstructive pulmonary disease. Am Rev Respir Dis, 1988; 138: 689-96

8. Britto RR, Rezende NR, Marinho KC et al: Inspiratory muscular training in chronic stroke survivors: a randomized controlled trial. Arch Phys Med Rehabil, 2011; 92: 184-90

9. Sutbeyaz ST, Koseoglu F, Inan L, Coskun O: Respiratory muscle training improves cardiopulmonary function and exercise tolerance in subjects with subacute stroke: a randomized controlled trial. Clin Rehabil, 2010; 24 240-50

10. Laroche CM, Moxham J, Green M: Respiratory muscle weakness and fatigue. Q J Med, 1989; 71: 373-97

11. Romer LM, McConnell AK: Inter-test reliability for non-invasive measures of respiratory muscle function in healthy humans. Eur J Appl Physiol, 2004; 91: 167-76

12. Larson JL, Covey MK, Vitalo CA et al: Maximal inspiratory pressure. Learning effect and test-retest reliability in patients with chronic obstructive pulmonary disease. Chest, 1993; 104: 448-53 size was relatively small. Further studies should be conducted with other patients who have impaired pulmonary function and weakened inspiratory muscles to investigate the effect of respiratory muscle training on pulmonary function.

\section{Conclusions}

Intra- and inter-rater reliability of this study confirm previous results and indicate that MIP, PIF, and VC can be reliably measured with an electronic inspiratory loading device, even by newly trained physical therapists. The smaller inter-rater reliability could be explained by the operator-dependence of this device. The potential use of electronic inspiratory loading device in clinical evaluation of the MIP, PIF, and VC remains unknown, but this study provides a basis for future research in clinical populations.

13. Kulnik ST, Birring SS, Moxham J et al: Does respiratory muscle training improve cough flow in acute stroke? Pilot randomized controlled trial. Stroke, 2015; 46: 447-53

14. Pinheiro MB, Polese JC, Faria CD et al: Inspiratory muscular weakness is most evident in chronic stroke survivors with lower walking speeds. Eur J Phys Rehabil Med, 2014; 50: 301-7

15. Fry DL, Hyatt RE: Pulmonary mechanics. A unified analysis of the relationship between pressure, volume and gasflow in the lungs of normal and diseased human subjects. Am J Med, 1960; 29: 672-89

16. Schneebeli A, Egloff M, Giampietro A et al: Rehabilitative ultrasound imaging of the supraspinatus muscle: Intra- and interrater reliability of thickness and cross-sectional area. J Bodyw Mov Ther, 2014; 18: 266-72

17. Black LF, Hyatt RE: Maximal respiratory pressures: normal values and relationship to age and sex. Am Rev Respir Dis, 1969; 99: 696-702

18. Neder JA, Andreoni S, Lerario MC, Nery LE: Reference values for lung func tion tests. II. Maximal respiratory pressures and voluntary ventilation. Braz J Med Biol Res, 1999; 32: 719-27

19. Bland JM, Altman DG: Statistical methods for assessing agreement between two methods of clinical measurement. Lancet, 1986; 1: 307-10

20. Rankin G, Stokes M: Reliability of assessment tools in rehabilitation: an illustration of appropriate statistical analyses. Clin Rehabil, 1998; 12: 187-99

21. Munro BH: Statistical methods for health care research. Lippincott Williams \& Wilkins, London UK. 2005

22. Roth EJ, Noll SF: Stroke rehabilitation. 2. Comorbidities and complications Arch Phys Med Rehabil, 1994; 75: S42-46

23. Fry DK, Pfalzer LA, Chokshi AR et al: Randomized control trial of effects of a 10-week inspiratory muscle training program on measures of pulmonary function in persons with multiple sclerosis. J Neurol Phys Ther, 2007; 31: 162-72

24. Fregonezi GA, Resqueti VR, Guell R et al: Effects of 8-week, interval-based inspiratory muscle training and breathing retraining in patients with generalized myasthenia gravis. Chest, 2005; 128: 1524-30

25. Xiao $\mathrm{Y}$, Luo $\mathrm{M}$, Wang J, Luo $\mathrm{H}$ : Inspiratory muscle training for the recovery of function after stroke. Cochrane Database Syst Rev, 2012; 5: CD009360

26. Gosselink R, Wagenaar RC, Decramer M: Reliability of a commercially available threshold loading device in healthy subjects and in patients with chronic obstructive pulmonary disease. Thorax, 1996; 51: 601-5 